\title{
Specialty Choice Stability: Are There Implications for Early Entry Into Residency?
}

Rebecca E. Cantone, MD | Nicole M. Deiorio, MD | Alex Polston, MD | Benjamin Schneider, MD

PRIMER. 2018;2:30.

Published: 12/12/2018 | DOI: 10.22454/PRiMER.2018.643028

\section{Abstract}

Introduction: The medical education community is piloting early entry to residency as a potential outcome to competency-based education and as a way to more quickly train future physicians in specialties of need. However, there is limited knowledge of which specialties may be best suited to this streamlined training. Student career desires may change over the course of their undergraduate training. We aimed to understand which specialties have stable student interest from matriculation to residency match in an effort to highlight which fields may be reasonable to consider for such accelerated programs.

Methods: Medical students at one school of medicine who matriculated in the years 2009-2013 were surveyed upon entry regarding the medical specialty they were most interested in pursuing. Six hundred fifty-four students were eligible for inclusion and 535 of the records met all requirements. On completion of medical school, final specialty choice for students obtaining a residency position was recorded. The data were analyzed to assess specialties with the highest versus the lowest rates of retention.

Results: Of 535 included students, the top specialties with retention of interest (no change in specialty choice for that student) from matriculation to match were physical medicine and rehabilitation, $(100 \%, \mathrm{n}=3$ retention/3 initial), psychiatry $(57.1 \%, 4 / 7)$, internal medicine $(48.5 \%, 47 / 97)$, and family medicine $(47.7 \%, 41 / 86)$. The specialties with the lowest retention were pathology $(0 \%, 0 / 2)$, preventive medicine $(0 \%, 0 / 4)$, dermatology $(12 \%, 1 / 8)$, neurology $(16.7 \%, 3 / 18)$ and radiation oncology $(16.7 \%, 1 / 6)$.

Discussion: Some specialties that attract student interest before matriculation may be more likely to maintain interest compared to others. This suggests a need for further research to determine if residency entry can begin earlier than traditionally thought, with certain fields better suited for accelerated training.

\section{Introduction}

Undergraduate medical education (UME) is continuing to evolve to prepare students for practice, with a growing focus on competency-based education. ${ }^{1,2}$ At some institutions, students may complete graduation requirements more quickly than in traditional, time-based curricula, and begin graduate medical education (GME) earlier. ${ }^{3}$ Some fields may maintain student interest from matriculation to match, while others may be more prone to fluctuation of interest during the UME experience. Understanding the stability of student specialty choice intent from start to finish of UME is important in order to confidently transition students into residency training sooner. On a larger scale, symptoms of burnout in residents have been associated with an elevated rate of specialty choice regret, ${ }^{4}$ which is a concerning finding if students are accelerated into specialties that have low stability across UME. There is currently a gap in understanding the stability of specialty choice across the UME experience. ${ }^{5}$

Retrospective data from residents ${ }^{6}$ and on retention of primary care interest in medical student graduates ${ }^{7,8}$ have 
been published, but recent studies do not focus on stability of specialty choice from start to finish of UME. There have been prior studies and reviews on specialty choice in medicine, ${ }^{9}$ factors associated with selecting primary care versus nonprimary care careers, ${ }^{10}$ and predictors of rural practice. ${ }^{11}$ However, the focus of this literature has primarily been on factors influencing ultimate specialty or practice choice, and not the student-level changes in interest from matriculation to match.

This question of congruency was assessed in the 1980's and data suggested that as high as 70\% of graduates' early choices remained stable. ${ }^{12}$ In that study, internal medicine (IM), family medicine (FM) and general surgery (GS) had the greatest interest at the start and end of medical school. Updated data are needed as at that time there were only 21 primary medical specialties ${ }^{13}$ versus the 26 in $2018,{ }^{14}$ and $62.5 \%$ of all US medical student graduates entered residency in IM, FM, or GS at that time versus $33 \%$ in 2018 . To address this gap in data, our key aim was to study the general and specialty-specific rate of interest stability from matriculation to match. We sought to guide accelerated UME training decisions with more updated data to examine if stability is more pronounced in certain specialties.

\section{Methods}

Our institutional review board approved all phases of this study. We retrospectively analyzed our UME program's prematriculation survey, a detailed survey sent to entering students in the school of medicine (SOM) in the weeks before their first day of medical school. The survey asks for the top anticipated specialty choice for each student, and results were accessed for those who matriculated from 2009-2013.

Six hundred and fifty-four students received the matriculation survey during that period and were eligible for inclusion in our data collection. Matriculated students were excluded if they did not complete the survey $(n=53)$, did not finish medical school $(n=19)$, were still enrolled as a student at the time of analysis $(n=38)$, or were admitted to the combined oral maxillofacial surgery program $(n=9)$.

Specialty choice was identified using the UME program's match data. Each student's initial and matched specialties were paired, then the data were deidentified for analysis. Data were grouped by matriculation year to account for differing times to complete the UME program. Each matriculating and graduating class was also sorted by specialty choice to calculate the percentage of each class interested in each specialty upon matriculation and graduation, respectively.

Each student record was classified as showing "retention" if the initial choice and match specialty were congruent. If interest diverged, we reported "recruitment" into the specialty in which they matched and "loss of interest" in the specialty they had initially chosen, maintaining awareness that loss of interest may also be related to perceived competitiveness to match in that field. Student records were sorted by specialty for each of the three groups to calculate percentages of retention, recruitment, and loss of interest. Specialties with less than one student at matriculation or match were also excluded. Because of the relatively small numbers of students who enter into surgical subspecialties each year, we placed the following specialties into a single group for analysis: cardiothoracic, neurologic, plastic, vascular, and urologic surgery, and otolaryngology.

Retention was calculated as the number of students matched into the specialty they chose at matriculation (numerator) divided by the total number of students who initially chose that specialty (denominator) and multiplied by 100 to report a percentage. Each student record was examined independently to discern individual changes from matriculation to match instead of overall percentages of the class. We also calculated percentages of recruitment and loss of interest to characterize specialties with lower retention rates. We selected $40 \%$ as a cutoff for greater retention based on a clear division in our data of specialties that had the highest rates, above the national retrospective average of $26 \%$ as reported by the AAMC. ${ }^{6}$

\section{Results}

Table 1 shows the retention data by specialty. Five hundred thirty-five students met the criteria for data analysis. The specialties of medical genetics, combined medicine-pediatrics, and nuclear medicine showed insufficient interest 
for analysis (less than one student each year) and were excluded. The mean retention across all specialties was $34.9 \%(187 / 535)$ and clustered grossly into three groups. Five specialties were high retainers at greater than $40 \%$, and eight specialties were low retainers at less than $20 \%$ (Figure 1), with the remainder of specialties falling in the middle. Physical medicine and rehabilitation had $100 \%$ retention, though the total number of students was small $(100 \%, n=3$ retention/3 initial). The next highest retaining specialties were psychiatry $(57.1 \%, 4 / 7)$, internal medicine $(48.5 \%, 47 / 97)$, family medicine $(47.7 \%, 41 / 86)$, and anesthesiology $(46.2 \%, 6 / 13)$; see Table 2 . The specialties with the lowest rates of retention were pathology $(0 \%, 0 / 2)$, preventive medicine $(0 \%, 0 / 4)$, dermatology $(12 \%, 1 / 8)$, neurology $(16.7 \%, 3 / 18)$ and radiation oncology $(16.7 \%, 1 / 6)$; see Table 3.

Table 4 reports specialties with the greatest increase in specialty interest during medical school while Table 5 reports those specialties with the highest loss of interest. These single-institution data provide the specialty interest stability from matriculation to match for $82 \%$ (535/653) of all students who matriculated in the years 2009-2013.

\section{Discussion}

These data suggest that specialty interest stability varies by specialty and that all specialties had students who lost and gained interest during the UME experience. This institution also shows a higher retention rate in specialty interest than the national average.

In considering the varying retention rates in certain fields, studies show that life experiences or additional extracurricular and degree experiences ${ }^{15,16}$ may factor into why students maintained the same specialty choice throughout medical school. For example, physical medicine and rehabilitation demonstrated $100 \%$ retention. Since this is likely a lesser-known specialty to the general population, we believe that students with interest in this at matriculation may have had premedical experience in this specialty. Despite commonly held beliefs, specialty interest groups may not increase specialty choice in a given field. ${ }^{17,18}$ Negative reputations of a specialty may also not impact specialty interest as previously thought. ${ }^{19-21}$ Control over lifestyle has recently been explored to account for shifts in specialty selection. ${ }^{22}$ More research should examine these concepts with student-specific data like ours to understand how students change their perception of fields throughout UME.

Limitations of this study are related to its single-site, pilot nature and the smaller numbers of students. The medical specialties break down into high, medium, and low stability groups; of the high stability specialties, only IM and FM have large enough numbers of total students to feel confident that these trends could be reproduced. Our additional retention specialties did not show the same volume of total student numbers during our study. Notably, final specialty choice as reported by match data may not reflect the actual desired specialty choice entering the match for students who were less competitive (or perceived as such) and therefore matched into a different field. Additionally, this single institution study was at the only university academic medical center in our state, and overt and hidden curricula that influence specialty choice may differ across other programs. A multicenter study would mitigate these limitations. However, historic specialty choice by percentage of the class from this medical school does mirror nationally reported rates, ${ }^{14}$ so we hope these findings may be reproducible at similar institutions.

As some institutions move toward a competency-based education system or consider accelerated entry into residency programs, we encourage more institutions to track these data. Based on our data, it may be wise to first target IM and FM for accelerated UME to GME pathways to prevent a loss of resources incurred if residents later change specialties or abandon the accelerated program. Institutions could lose significant financial resources if residents left an accelerated program due to decision regret. This study may suggest a need for programs to ensure students have opportunities for robust career exploration prior to participating in accelerated residency programs, as we do not know when these decision changes occur.

Finally, if student- or specialty-specific factors can be determined, it would be beneficial to use this information to target medical school matriculants who will eventually meet the workforce needs or demands of the health care system at the regional or national level. If specialty interest can be retained, targeted recruitment can be considered even prior to UME matriculation. 


\section{Conclusions}

Pilot data at one UME institution show a method and a need to examine which specialties have more persistent medical student interest from matriculation to graduation. This would assist in deciding which programs may be best suited for accelerated tracks into residency specialties. It would also assist in understanding student- or institution-specific factors that may contribute to the decision and allow for additional ideas in sustaining an appropriately balanced medical workforce.

\section{Tables and Figures}

Table 1: Specialty Choice Retention by Specialty

\begin{tabular}{|c|c|c|c|c|}
\hline Specialty (alphabetically) & $\begin{array}{l}\text { No. Selected at } \\
\text { Matriculation }\end{array}$ & $\begin{array}{l}\text { No. } \\
\text { Matched }\end{array}$ & $\begin{array}{l}\text { No. } \\
\text { Retention }\end{array}$ & $\begin{array}{l}\text { Percent } \\
\text { Retention }\end{array}$ \\
\hline Anesthesiology & 13 & 35 & 6 & $46.15 \%$ \\
\hline Dermatology & 8 & 2 & 1 & $12.50 \%$ \\
\hline Diagnostic radiology & 11 & 20 & 4 & $36.36 \%$ \\
\hline Emergency medicine & 72 & 58 & 25 & $34.72 \%$ \\
\hline Family medicine & 86 & 92 & 41 & $47.67 \%$ \\
\hline Internal medicine & 97 & 109 & 47 & $48.45 \%$ \\
\hline Medicine-pediatrics & 0 & 4 & 0 & - \\
\hline Neurology & 18 & 6 & 3 & $16.67 \%$ \\
\hline No match* & - & 6 & - & - \\
\hline Obstetrics and gynecology & 30 & 35 & 11 & $36.67 \%$ \\
\hline Ophthalmology & 6 & 10 & 2 & $33.33 \%$ \\
\hline Orthopedic surgery & 32 & 8 & 6 & $18.75 \%$ \\
\hline Pathology & 2 & 5 & 0 & $0.00 \%$ \\
\hline Pediatrics & 71 & 41 & 20 & $28.17 \%$ \\
\hline Physical medicine and rehabilitation & 3 & 5 & 3 & $100.00 \%$ \\
\hline Preliminary/transitional only & 0 & 14 & 0 & - \\
\hline Preventive medicine & 4 & 0 & 0 & $0.00 \%$ \\
\hline Psychiatry & 7 & 17 & 4 & $57.14 \%$ \\
\hline Radiation oncology & 6 & 6 & 1 & $16.67 \%$ \\
\hline Surgery-general & 37 & 41 & 7 & $18.92 \%$ \\
\hline $\begin{array}{c}\text { Surgical subspecialty: cardiothoracic, otolaryngology, } \\
\text { neurologic, plastic, urologic, vascular }\end{array}$ & 32 & 21 & 6 & $18.75 \%$ \\
\hline Total/Mean: & 535 & 535 & 187 & $34.95 \%$ \\
\hline
\end{tabular}

* Collected for data completion but not analyzed further. 
Figure 1: Percent Specialty Retention From Matriculation to Match

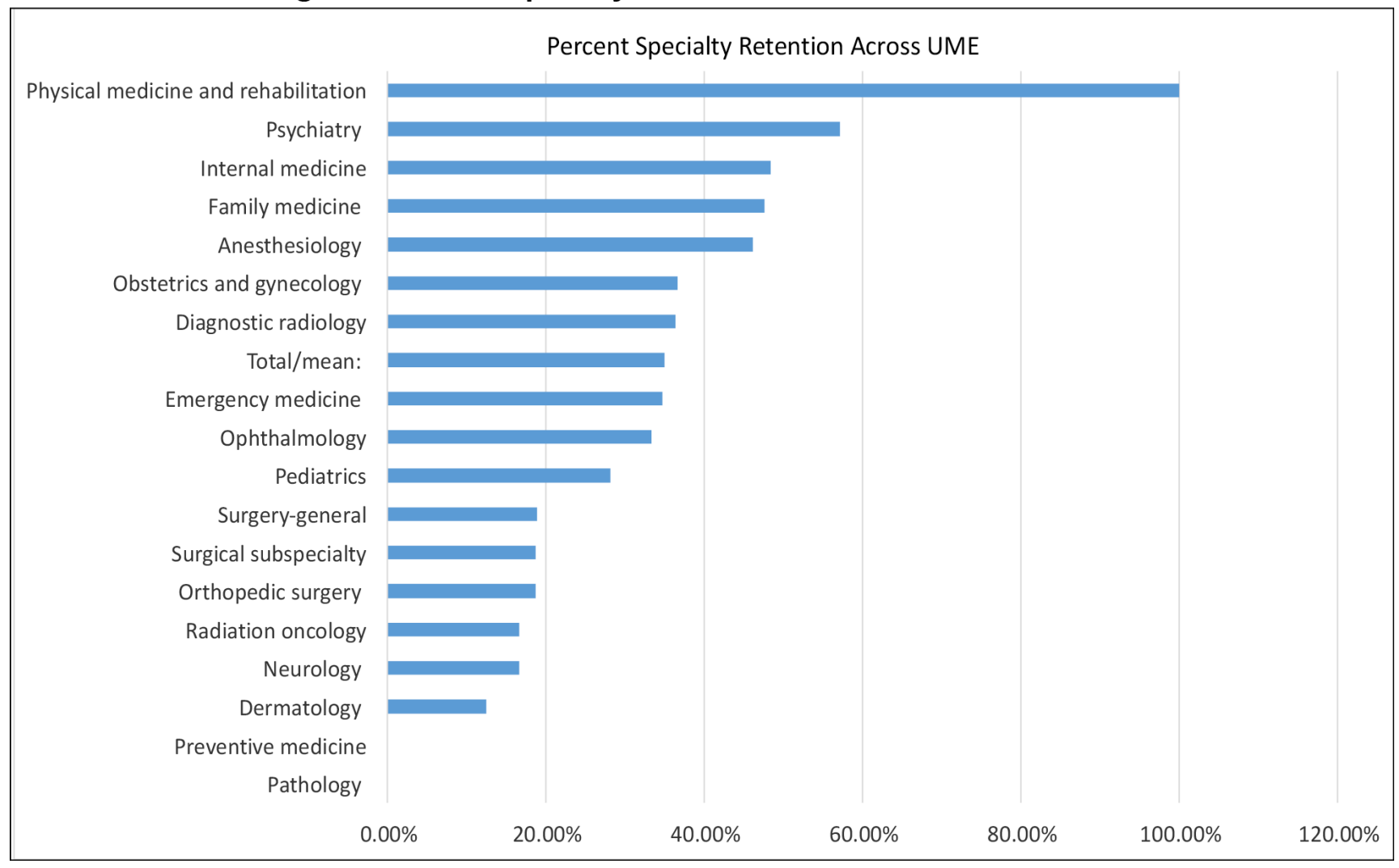

Table 2: Highest Retention Specialty Choices

\begin{tabular}{|c|c|}
\hline Specialty Choice & Percent Retention (n) \\
\hline Physical medicine and rehabilitation & $100.0(3)$ \\
\hline Psychiatry & $57.1(4)$ \\
\hline Internal medicine & $48.5(47)$ \\
\hline Family medicine & $47.7(41)$ \\
\hline Anesthesiology & $46.2(6)$ \\
\hline
\end{tabular}

Table 3: Lowest Retention Specialty Choices

\begin{tabular}{|c|c|}
\hline Specialty Choice & Percent Retention (n) \\
\hline Surgery-general & $18.9(7)$ \\
\hline Orthopedic surgery & $18.8(6)$ \\
\hline Surgical subspecialty & $18.8(6)$ \\
\hline Neurology & $16.7(3)$ \\
\hline Radiation oncology & $16.7(1)$ \\
\hline Dermatology & $12.5(1)$ \\
\hline Pathology & $0.0(0)$ \\
\hline Preventive medicine & $0.0(0)$ \\
\hline
\end{tabular}


Table 4: Recruitment Specialty Choices

\begin{tabular}{|c|c|}
\hline Specialty Choice & Percent Recruited In (n) \\
\hline Radiation oncology & $83.3(5)$ \\
\hline Surgery-general & $82.9(34)$ \\
\hline Anesthesiology & $82.9(29)$ \\
\hline Diagnostic radiology & $80.0(16)$ \\
\hline Ophthalmology & $80.0(8)$ \\
\hline Psychiatry & $76.5(13)$ \\
\hline
\end{tabular}

Table 5: Loss of Interest Specialty Choices

\begin{tabular}{|c|c|}
\hline Specialty Choice & Percent Changed Out $(\mathrm{n})$ \\
\hline Pathology & $100.0(2)$ \\
\hline Preventive medicine & $100.0(4)$ \\
\hline Dermatology & $87.5(7)$ \\
\hline Neurology & $83.3(15)$ \\
\hline Radiation oncology & $83.3(5)$ \\
\hline Orthopedic surgery & $81.3(26)$ \\
\hline Surgical subspecialty & $81.3(26)$ \\
\hline Surgery-general & $81.1(30)$ \\
\hline
\end{tabular}

\section{Acknowledgments}

The authors thank Anita D. Taylor for her creation of the prematriculation survey and maintenance of the records.

\section{Presentations:}

Polston A, Cantone R, Deiorio N, Schneider B. Specialty choice stability: are there implications for advising and early entry into residency? OHSU Educational Symposium, April 27, 2018.

Cantone R, Schneider B, Deiorio N. Medical student specialty choice: congruency from pre-matriculation to match. 2018 AAMC Annual Meeting - Learn Serve Lead. Highlights in Medical Education. Austin Texas, November 2-6, 2018.

\section{Corresponding Author}

\section{Rebecca E. Cantone, MD}

Oregon Health \& Science University, Mail code FM, 3181 SW Sam Jackson Parkway, Portland, OR 97239 cantone@ohsu.edu

\section{Author Affiliations}

Rebecca E. Cantone, MD - Department of Family Medicine, Oregon Health \& Science University, Portland, OR

Nicole M. Deiorio, MD - Virginia Commonwealth University School of Medicine, Richmond, VA

Alex Polston, MD - Alaska Family Medicine Residency, Anchorage, AK

Benjamin Schneider, MD - Department of Family Medicine, Oregon Health \& Science University, Portland, OR

\section{References}

1. Abramson SB, Jacob D, Rosenfeld M, et al. A 3-year M.D.-accelerating careers, diminishing debt. N Engl J 
Med. 2013;369(12):1085-1087. https://doi.org/10.1056/NEJMp1304681

2. Andrews JS, Bale JF Jr, Soep JB, et al; EPAC Study Group. Education in pediatrics across the continuum (EPAC): first steps toward realizing the dream of competency-based education. Acad Med.

2018;93(3):414-420. https://doi.org/10.1097/ACM.0000000000002020

3. Hauer KE, Durning SJ, Kernan WN, et al. Factors associated with medical students' career choices regarding internal medicine. JAMA. 2008;300(10):1154-1164. https://doi.org/10.1001/jama.300.10.1154

4. Dyrbye LN, Burke SE, Hardeman RR, et al. Association of clinical specialty with symptoms of burnout and career choice regret among US resident physicians. JAMA. 2018;320(11):1114-1130. https://doi.org/10.1001 /jama.2018.12615

5. Wright B, Scott I, Woloschuk W, Brenneis F, Bradley J. Career choice of new medical students at three Canadian universities: family medicine versus specialty medicine. CMAJ. 2004;170(13):1920-1924. https://doi.org/10.1503/cmaj.1031111

6. Association of American Medical Colleges. Report on Residents, Data \& Analysis Table A1: Continuity of Specialty Preference on the Matriculating Student Questionnaire and the Graduation Questionnaire. https://www.aamc.org/data/484712/report-on-residents-2017-a1table.html. Accessed December 7, 2018.

7. Compton MT, Frank E, Elon L, Carrera J. Changes in U.S. medical students' specialty interests over the course of medical school. J Gen Intern Med. 2008;23(7):1095-1100. https://doi.org/10.1007/s11606-008-0579-z

8. Zeldow PB, Preston RC, Daugherty SR. The decision to enter a medical specialty: timing and stability. Med Educ. 1992;26(4):327-332. https://doi.org/10.1111/j.1365-2923.1992.tb00177.x

9. Bland CJ, Meurer LN, Maldonado G. Determinants of primary care specialty choice: a non-statistical metaanalysis of the literature. Acad Med. 1995;70(7):620-641. https://doi.org/10.1097 /00001888-199507000-00013

10. Bennett KL, Phillips JP. Finding, recruiting, and sustaining the future primary care physician workforce: a new theoretical model of specialty choice process. Acad Med. 2010;85(10)(suppl):S81-S88. https://doi.org /10.1097/ACM.0b013e3181ed4bae

11. Rabinowitz HK, Diamond JJ, Markham FW, Santana AJ. The relationship between matriculating medical students' planned specialties and eventual rural practice outcomes. Acad Med. 2012;87(8):1086-1090. https://doi.org/10.1097/ACM.0b013e31825cfa54

12. Carline JD, Greer T. Comparing physicians' specialty interests upon entering medical school with their eventual practice specialties. Acad Med. 1991;66(1):44-46. https://doi.org/10.1097 /00001888-199101000-00013

13. National Resident Matching Program. 1986 NRMP Data. https://mkOnrmpcikgb8jxyd19h.kinstacdn.com/wpcontent/uploads/2013/08/resultsanddata1986.pdf. Accessed December 7, 2018.

14. National Resident Matching Program. Results and Data Main Residency Match. (2016). http://www.nrmp.org /wp-content/uploads/2016/04/Main-Match-Results-and-Data-2016.pdf. Accessed December 7, 2018.

15. Kost A, Cawse-Lucas J, Evans DV, Overstreet F, Andrilla CHA, Dobie S. Medical student participation in family medicine department extracurricular experiences and choosing to become a family physician. Fam Med. 2015;47(10):763-769.

16. Brown A, Ismail R, Gookin G, Hernandez C, Logan G, Pasarica M. The effect of medical student volunteering in a student-run clinic on specialty choice for residency. Cureus. 2017;9(1):e967. https://doi.org/10.7759 /cureus. 967

17. McKee ND, McKague MA, Ramsden VR, Poole RE. Cultivating interest in family medicine: family medicine interest group reaches undergraduate medical students. Can Fam Physician. 2007;53(4):661-665.

18. Baraka SM, Ebell MH. Family medicine interest groups at US medical schools. Fam Med. 1995;27(7):437-439.

19. Burack JH, Irby DM, Carline JD, Ambrozy DM, Ellsbury KE, Stritter FT. A study of medical students' specialtychoice pathways: trying on possible selves. Acad Med. 1997;72(6):534-541. https://doi.org/10.1097 /00001888-199706000-00021

20. Hunt DD, Scott C, Zhong S, Goldstein E. Frequency and effect of negative comments ("badmouthing") on medical students' career choices. Acad Med. 1996;71(6):665-669. https://doi.org/10.1097 /00001888-199606000-00022

21. Rohan-Minjares F, Alfero C, Kaufman A. How Medical Schools Can Encourage Students' Interest in Family 
Medicine. Acad Med. 2015;90(5):553-555. https://doi.org/10.1097/ACM.0000000000000569

22. Dorsey ER, Jarjoura D, Rutecki GW. Influence of controllable lifestyle on recent trends in specialty choice by US medical students. JAMA. 2003;290(9):1173-1178. https://doi.org/10.1001/jama.290.9.1173

Copyright $\odot 2018$ by the Society of Teachers of Family Medicine 